\title{
Numerical Simulations on Nonlinear Dynamics in Lasers as Related High Energy Physics Phenomena
}

\author{
Andreea Rodica Sterian \\ Academic Center of Optical Engineering and Photonics, Polytechnic University of Bucharest, 313 Splaiul Independentei, \\ 060042 Bucharest, Romania \\ Correspondence should be addressed to Andreea Rodica Sterian; andreea.sterian1@yahoo.com
}

Received 4 August 2013; Accepted 16 September 2013

Academic Editor: Mehmet Bektasoglu

Copyright (C) 2013 Andreea Rodica Sterian. This is an open access article distributed under the Creative Commons Attribution License, which permits unrestricted use, distribution, and reproduction in any medium, provided the original work is properly cited.

\begin{abstract}
This paper aims to present some results on nonlinear dynamics in active nanostructures as lasers with quantum wells and erbium doped laser systems using mathematical models, methods, and numerical simulations for some related high energy physics phenomena. We discuss nonlinear dynamics of laser with quantum wells and of fiber optics laser and soliton interactions. The results presented have important implications in particle detection and postdetection processing of information as well as in soliton generation and amplification or in the case that these simulations are thought to be useful in the experiments concerning the high energy particles. The soliton behaviour as particle offers the possibility to use solitons for better understanding of real particles in this field. The developed numerical models concerning nonlinear dynamics in nanostructured lasers, erbium doped laser systems, the soliton interactions, and the obtained results are consistent with the existing data in the literature.
\end{abstract}

\section{Introduction}

Numerical simulations on the nonlinear dynamics in lasers are largely presented in the literature, but the research work for their development has determined unprecedented progress in particle physics [1-8].

The research put into evidence the existence of relevant conditions and limitations which are important in the particle detectors and data processing after detection and in the experimental studies in high energy physics also. These unprecedented advances offer opportunities in theoretical and numerical predictions in high energy physics and also in the related fields. The particles having soliton behavior, as being localized and preserved under collisions, lead to a large number of applications but offer the possibility to use solitons for better understanding of the real particles, in high energy physics. On the other hand, the research developing in Romania concerning the Project "Extreme Light InfrastructureNuclear Physics" (ELI-NP) demonstrate the complexity of problems in the physics and engineering of high energy particles [9]. The ELI-NP facilities, imply as main tools for the research directions, and the realization of the laser systems of high power of petawatt and ultrashort pulses together with a gamma beam system, highly collimated and very brilliant and tunable $[10,11]$, so that numerical simulations on nonlinear dynamics are essential and inevitable.

The mathematical models, the methods, and the numerical simulations from nonlinear dynamics are important in processing of data concerning particle physics. Some numerical results concerning the dynamics of a two-level medium under the action of short optical pulses are presented in the papers [12-18]. We analyse in the paper the nonlinear dynamics of the lasers with quantum wells and optic fibers as well as the soliton collisions and the behavior of particles in high energy physics as solitons. The used methods are typical for the study of quantum fields and particles, particularly cosmic rays [19].

\section{Modulation Capabilities and Bifurcation Diagrams}

The numerical simulations of the lasers with quantum wells (QWL) are based on solving the nonlinear differential equations which describe the behaviour of this kind of 


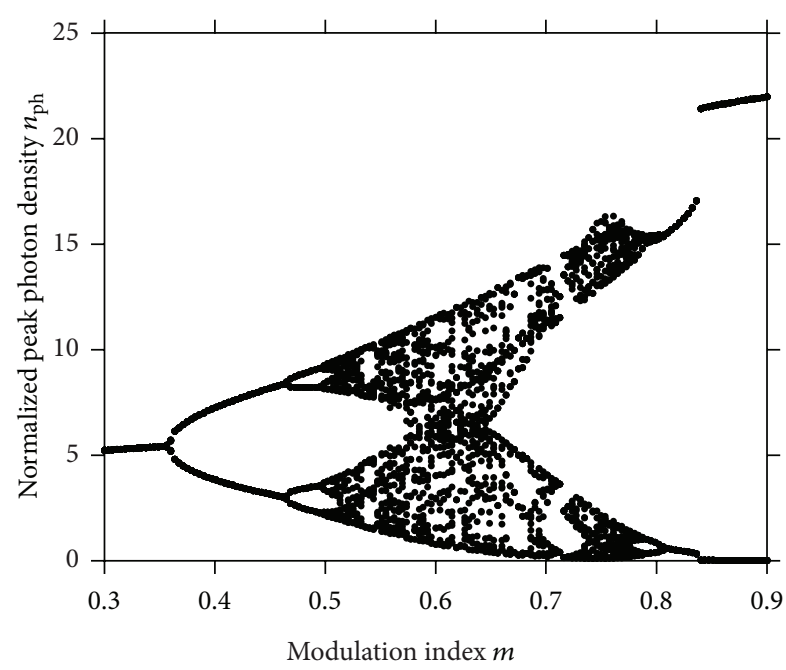

FIGURE 1: Bifurcation diagram: photon density dependence of modulation index.

devices [20-31]. Study of functional characteristics of QWL is possibly based on the models present in [20], by implementing programs to integrate these equations in MathCad using appropriate algorithms. The MathCad software has the advantage of solving complicated systems starting from the usual mathematical form by comparison with other methods of integration of the nonlinear equations. The modulation studies are important, and for solitons generation in optical fibers also, the phase self-modulation phenomena are the essential mechanism of this process [26]. In general, all the analysed models had given satisfactory results for the study of transient and dynamics operational regimes at the small signal of injection. At high level of injection, the obtained numerical results had showed the limits of specific model used but are consistent with the theoretical analysis.

We have considered the amplitude modulation of the current of injection [27]. The output power of the laser versus the modulation index was investigated (Figure 1).

Graphical representation of the modulated signal simultaneously with the evolution of the system in the phase space highlights also the route to chaos with the increase of $m$ by doubling the period.

We have examined the diagram of bifurcation of the photon density versus the modulation index directly (with the increase of $m$ ) and vice versa (with decreasing of $m$ ) for a cycle of modulation as an indicator of the system progress. The chaotic region has periodic windows and a dynamic type of hysteresis.

We have considered also the additional noise sources in the rate equations [28]. In the Markov approximation, the noise sources are random Gaussian variables with zero mean and delta-correlated quantities [24]. For numerical representation of the dynamics including the noise sources, we had used the random Gaussian numbers applied by MATLAB functions. Numerical calculations showed that a small noise disturbs and can also occur transitions between coexisting attractors (Figure 2).
For a suitable modulation index, there are two coexisting stable attractors as it is showed in Figures 2(a) and 2(b), but in the presence of the noise, the induced transitions from one attractor to another can occur (Figure 2(c)). The contribution of the included noise had proved to be an important factor that limits the design of the format of modulation.

\section{Case Study on Nonlinear Dynamics}

A case study in nonlinear dynamics of lasers with quantum wells is analysed systematically based on the model with two equations proposed by Anghel et al. [29-31]. These equations were solved numerically using typical parameters of a laser with multiple quantum wells. The parameter values used in numerical simulations are presented in the papers [20, 21]. Simulations were performed in MATLAB programming language using a scheme Runge-Kutta of order four. Detailed numerical investigations showed that for the lower frequency compared to the frequency of relaxation, dynamics of the system is periodic with the period of the radio-frequency modulating signal.

For a sufficiently low modulation index is obtained a sinusoidal periodic regime, so that laser output power is sinusoidal. For a growth of the modulation index and the frequency of modulation near the frequency of the relaxation oscillations, a pulsing mode of operation is obtained with a duration of tens of picoseconds (ultrashort pulses), so that the laser system can be used as a source of pulses for optical communications. Higher frequency modulation causes multiple bifurcation points including a chaotic dynamics for certain parameter values (Figure 3). Also, in Figure 3 critical dependence of the modulation index (which leads to the bifurcation) of frequency is observed.

The behavior is similar for other values of the injection current above the threshold. However, an increasing dependence of the modulation index for the doubling of period with the current by the polarization can be observed.

\section{Nonlinear Dynamics in Optical Fibers Systems}

In recent years much attention has been paid to the study of nonlinear dynamics in optical fiber lasers [32-39].

Numerical simulations discussed here refer to nonlinear effects in such kind of systems. The chaotic dynamics was studied numerically using the rate equations, based on phenomenon of formation of the ion pairs [40-42]. It was demonstrated the stable, nonchaotic operation of the analyzed laser system and discussed the performance of their modulation using the methods of communications theory.

Numerical modeling was realized using the MATLAB programming medium. The base element of the program was the function ode 45 , which realize the integration of the right side expressions of the nonlinear coupled equation using the method Runge-Kutta. The self-pulsing and chaotic functioning of the EDFLs have been reported in various experimental conditions including in the case the pumping is near the laser threshold [32-35]. A model for the single-mode 


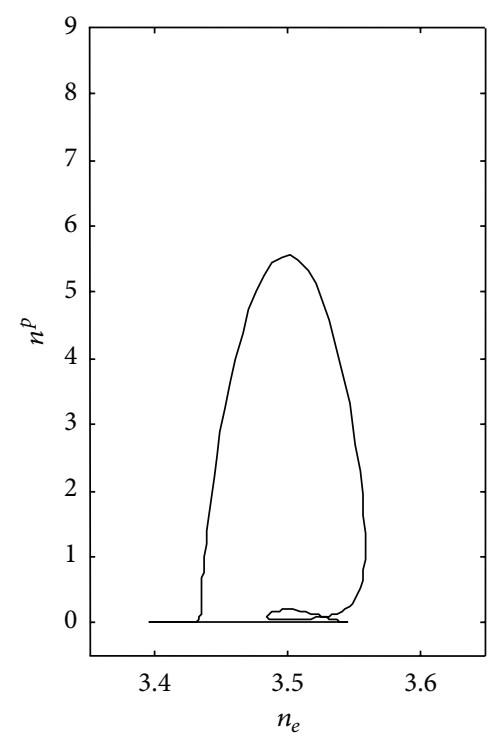

(a)

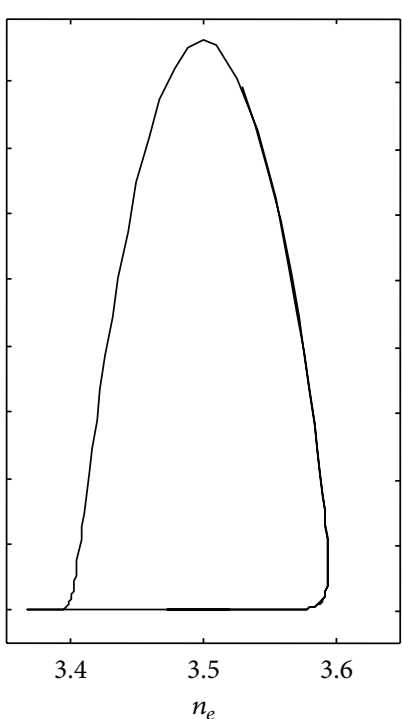

(b)

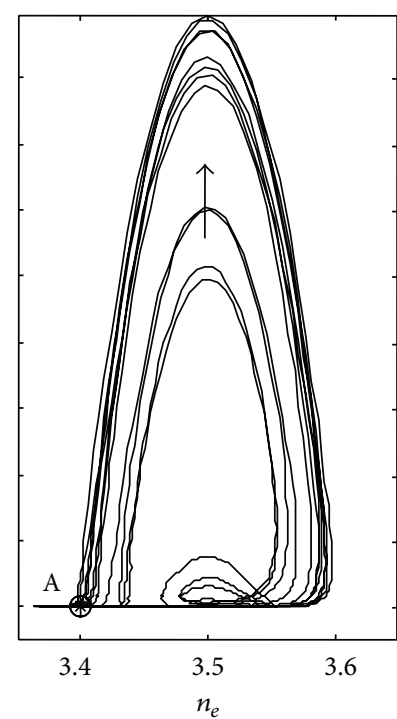

(c)

FIGURE 2: Gaussian noise influence on the system evolution in the phase plane.

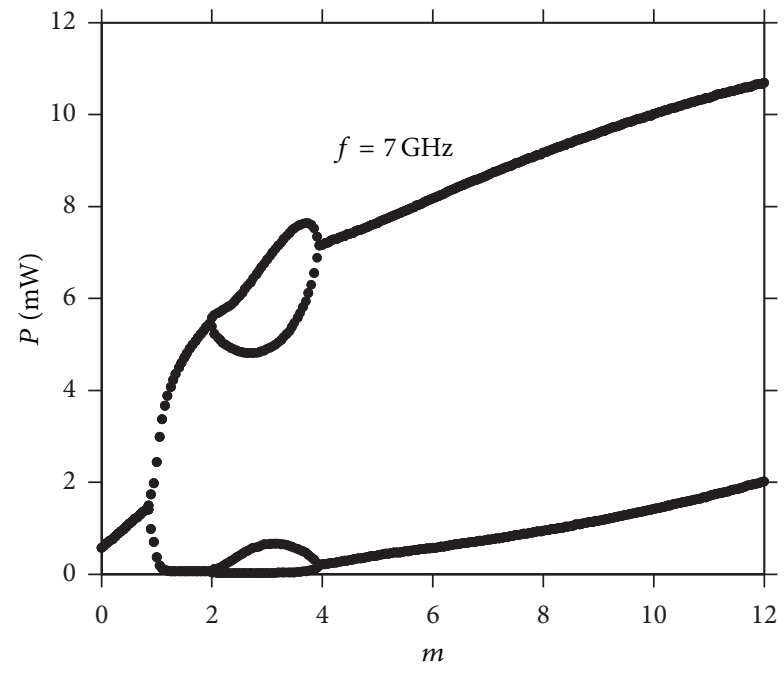

(a)

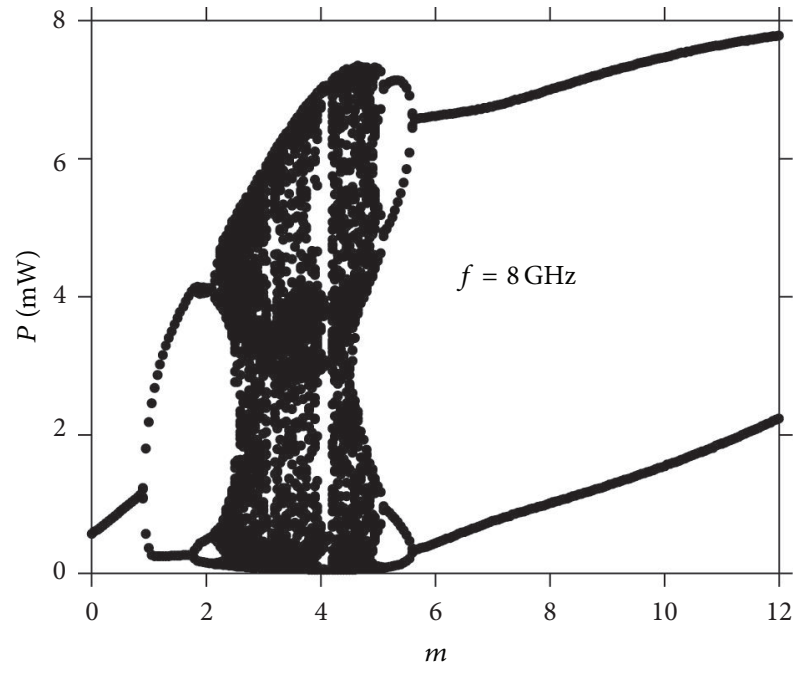

(b)

FIgURE 3: The laser bifurcation diagrams for different modulation frequencies.

laser was analysed firstly taking into account the presence of the erbium ion pairs that act as a saturable absorber. For this type of laser system don't yet completely are known the interaction mechanisms, in spite of many published works. The nonlinear dynamics of an erbium doped fiber laser is explained based on a simple model of the ion pairs present in heavily doped fibers. The dynamics is reducible to four nonlinear differential equations. Next, the nonlinear dynamics of a two-mode laser with optical fiber is explained in terms of a classical two-modes laser model and two additional equations of the quantum states of ion pairs [40], so that the laser dynamics is described through six coupled differential equations. At a suitable level of the concentration of the ion pairs, the laser system is in a steady state or selfpulsing operation regime, depending on the value of the pumping parameter. For larger values of the ion pairs, there is a range of self-pulsation dynamics. This is showed by means of a bifurcation diagram in which the mode intensity of the laser is plotted against the pumping parameter. This dynamics has also been encountered in the case of the one-mode model, the change from a stationary stable state to a self-pulsing one being a Hopf bifurcation.

Figure 4(a) shows the maxima of intensity at a larger concentration of the ion pairs. There exists a region of two maximum intensities, that is, a period doubling. A small increase of the ion pairs concentration (Figure 4(b)) leads 


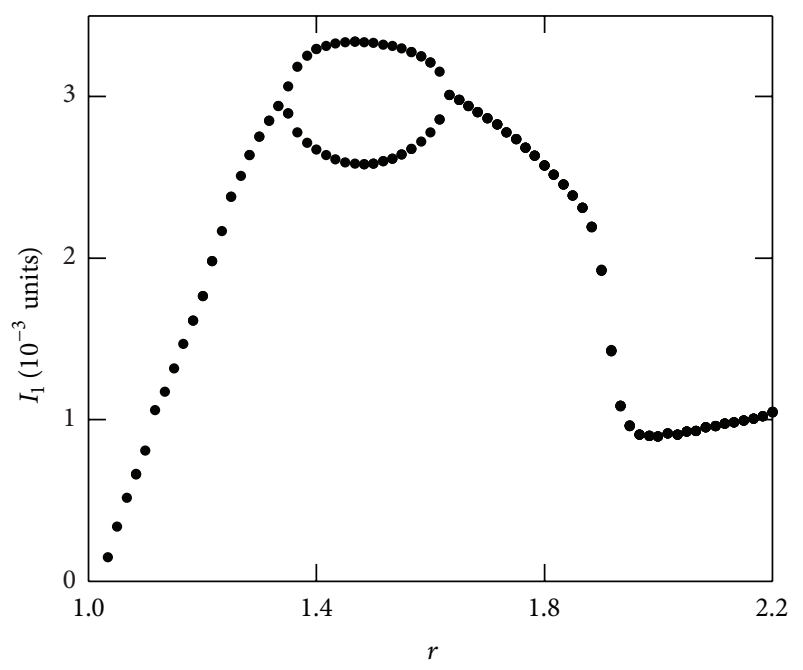

(a)

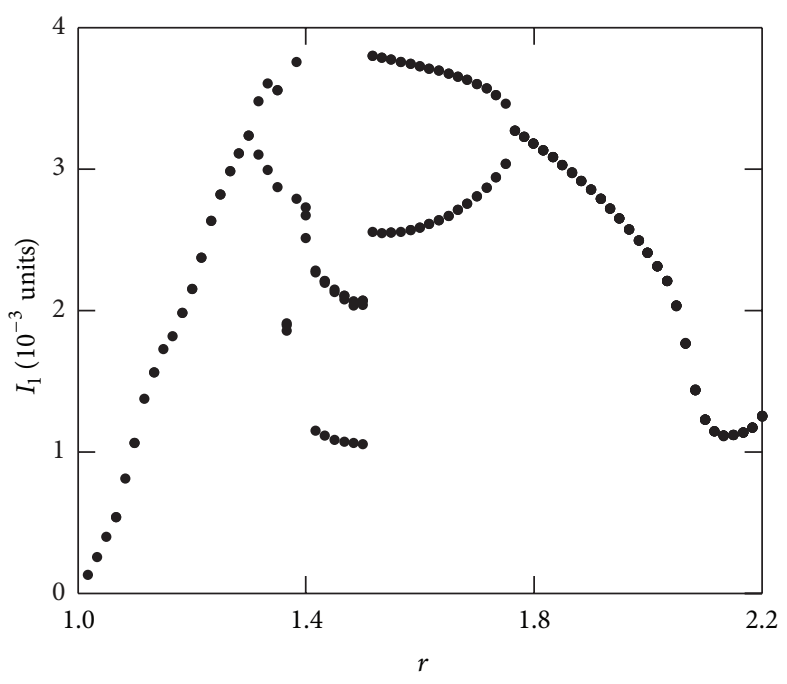

(b)

FIGURE 4: Bifurcation diagram of the mode intensity for two values of ion pairs versus the pumping strength $r$.

to the appearance of a window of a tripled period. Further increase of the ion-pairs concentrations leads to a more complicated bifurcation diagram, including of the regions with multiple stable states (generalized bistability) and routes to chaotic dynamics such as the period-doubling route or a quasiperiodic one.

The used method of the fourth-order Runge-Kutta for the numerical simulation demonstrate the importance of "computer experiments" in the designing, improving, and optimization of these coherent optical systems.

Some new features of the computer analysed systems and the existence of some new situations have been put into evidence for their use by the designers in different applications [42-48]. The obtained results are important not only for the optimization of the functioning conditions of this kind of devices but also for designing of the systems for the processing of data in the particle physics and in the systems engineering of high energy.

\section{Numerical Simulation of Soliton Propagation}

In the paper [49] the specific features of the soliton pulses were studied, so: the type of the nonlinearity, dispersive properties of the propagation medium and the propagation equations, for the optical and acoustic solitons. Were analysed the specific equations as quadratic nonlinear Schrodinger (QNLS), Kerr cubic (CNLS), Saturable (SNLS), discretized (DNLS), Korteveg de Vries (KdV), Boussinesq, Burgers, and sine-Gordon. Also a study of the methods of discretization used by the different numerical simulations was realised.

A numerical simulation of soliton propagation, based on the Korteweg-de Vries equation, using a powerful PC program (Maple10) which permits to perform numerical calculations, plot or animate functions, and manage analytical expressions is presented in paper [50]. We discuss a model of the interaction between two solitons, taking into account both the fiber dispersion and nonlinearity. The numerical simulations show how the solitons are propagating in optical fibers and how two solitons interact, passing one through another, with only a phase change. These simulations are thought to be useful for the designers working in the digital data transmission and in particles physics as well. The program Maple10 helps us plot solutions (we can use animation or individual frames) for different values of the wave number $k$, for different moments $t$, and for an arbitrary interval for $x$. For the study of the interaction between two solitons, we need a solution of the KdV equation present in $[3,50]$ :

$$
\begin{aligned}
& u 2:=2\left(-k_{2}^{2} k_{1}^{2} \cosh \left(k_{1}\left(x-4 k_{1}^{2} t\right)\right)^{2}\right. \\
& +k_{2}^{2} k_{1}^{2} \cosh \left(k_{2}\left(x-4 k_{2}^{2} t\right)\right)^{2} \\
& \quad-k_{2}^{2} k_{1}^{2}-k_{1}^{4} \cosh \left(k_{2}\left(x-4 k_{2}^{2} t\right)\right)^{2} \\
& \left.+k_{1}^{4}+\cosh \left(k_{1}\left(x-4 k_{1}^{2} t\right)\right)^{2} k_{2}^{4}\right) \\
& \times\left(\quad \cosh \left(k_{1}\left(x-4 k_{1}^{2} t\right)\right) \cosh \left(k_{2}\left(x-4 k_{2}^{2} t\right)\right) k_{2}\right. \\
& \quad-\sinh \left(k_{2}\left(x-4 k_{2}^{2} t\right)\right) \\
& \left.\left.\quad \times \sinh \left(k_{1}\left(x-4 k_{1}^{2} t\right)\right) k_{1}\right)^{2}\right)^{-1} .
\end{aligned}
$$

With that solution, Maple10 helps us visualize the interaction between the two solitons as in Figure 5. 

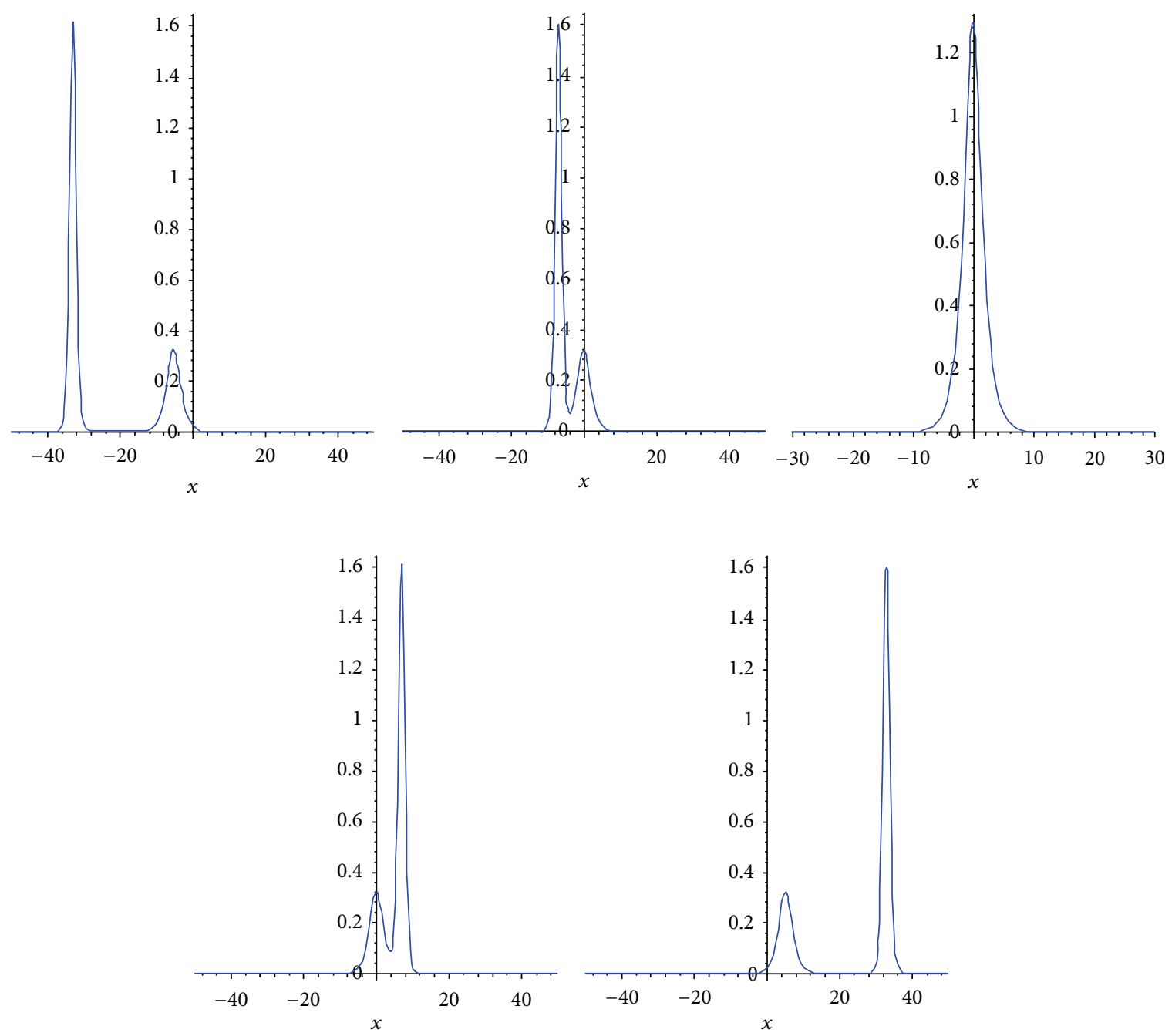

Figure 5: The interaction of two solitons for $k_{1}=0.4 ; k_{2}=0.9 ; x=-50 \cdots 50 ; t=-10 ;-2 ; 0 ; 2 ; 10$.

We can see at both solitons the regaining of their shapes after collision (after they pass one through another). Obviously, it is better to use the animation feature of the Maple10 program by modification of the parameters and observing the changes.

For designers working in digital data transmission, the simulation of the solitons propagation along optical fibers is useful and may be performed successfully also with Maple10. The animated images are some useful features of this computer program. Especially, the collision of two solitons and of $k$ dependence of the soliton propagation can be studied by this means, respectively.

As it was stressed above, after the collision, the solitons regain their original shape and velocity $[8,50,51]$. The only remaining effect of the scattering is a phase shift (i.e., a change in the position they would have reached without interaction). For the trisolitonic model, using Maplell similarly, the solution is plotted in Figure 6, showing the collision of three solitons $[49,51]$, by choosing various values for the parameters.

\section{Solitons Like Behavior of Particles in High Energy Physics}

The behavior as particle of the solitons (they are localised and preserved in the collision) leads to a large number of applications and is important in the particle physics also [50]. It is well known that we hope the solitons to be used for a better understanding of real particles. There are already soliton models of nuclei. On the other hand, there are theories which permit us to see particles such as electrons and positrons as being solitons in appropriate cases. In the paper [52] they had proposed a general semiclassical method for computing of the probability of the production of solitonantisoliton pairs in the particle collisions. They calculated also the probability of this process at high energies. In the paper [53] they had studied the creation of the solitons from the particles, using an suitable model used as a prototype. It was considered the scattering of the small identical wave pulses, which are equivalent to a sequence of particles, and they found that kink-antikink pairs are created in a large region of the parameters space. The dissertation [54] is devoted 

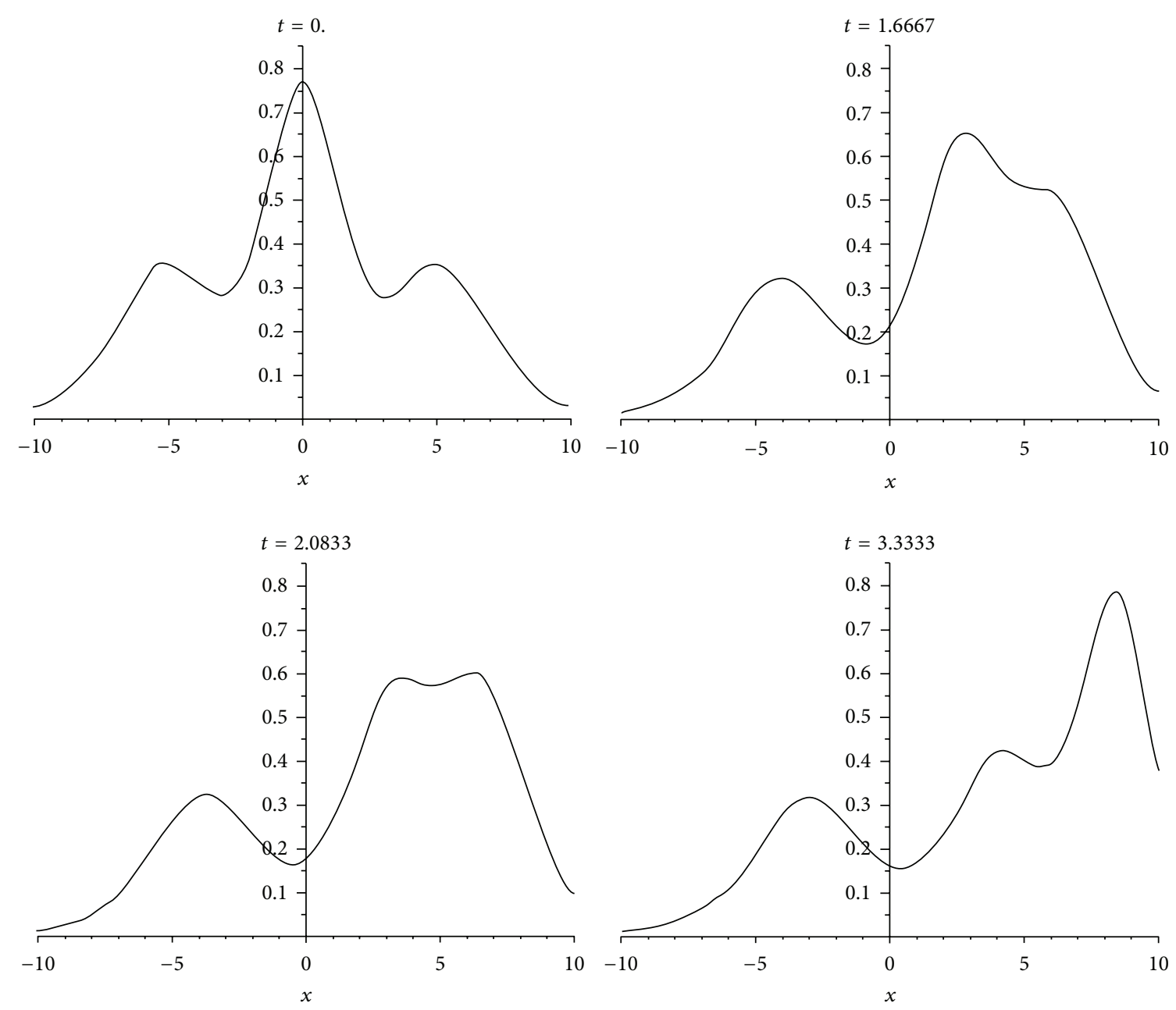

FIGURE 6: The interaction of three solitons.

to the study of monopoles and solitons in particle physics. The monopoles and solitons are of relevance as intermediate and final states of the particles in the collision experiments. The study of monopoles and solitons in the theory of strong interactions had shown the possible interpretation of the solitons as baryons. However, so far there is no general theory in which the particles are described as solitons. There are macroscopic phenomena such as the spontaneous transparency and the behavior of light in fiber optics that are understood only in terms of solitons. The methods specified above are typical for the quantum fields and the particles study, particularly of the cosmic rays [55-61].

\section{Conclusions}

(i) The nonlinear dynamics in lasers was treated taking into account the complexity of the phenomena which govern the field-substance interaction with applications in particle detectors and data processing after detection, but these methods are typical for the study and for quantum fields and particles also. (ii) A computational software was elaborated to study the nonlinear phenomena in quantum well lasers (QWL). Mathcad proved to be a powerful tool in analyzing and designing of these models. For the QWL, we put into evidence their complex nonlinear dynamics, characterized by bifurcation points and chaos, the critical values of the parameters being determined.

(iii) The classical Runge-Kutta method was used successfully for numerical simulation of the complex nonlinear dynamics in lasers with optical fibers. The chaotic dynamics was studied numerically based on the rate equations and on the phenomenon of formation of the ion pairs.

(iv) Our results put into evidence the existence of the new situations which are important for the optimization of the functioning conditions for this kind of devices. The nonlinear dynamics can be controlled, and undesired situations can be avoided if it is necessary, by suitable parameters choosing. 
(v) The studies on laser modulation are important for the generation of solitons in optical fibers, the phase selfmodulation phenomenon being the essential mechanism of this process. The study of the interaction between two solitons using a powerful PC program (Maple10) is of great interest for a better understanding of real particles in spite of the fact that now there is no general theory in which the particles are described as solitons.

(vi) The developed numerical methods and the obtained results are consistent with the existing data in the literature. The methods of the computer experiments make a complex study possible taking into account the intercorrelations of the parameters by simulating experimental conditions, as it has been shown, so that the obtained results can be successfully applied for processing of data in the particle physics.

\section{Conflict of Interests}

The author declares that there is no conflict of interests regarding the publication of this paper.

\section{References}

[1] G. P. Agrawal, Nonlinear Fiber Optics, Academic Press, San Diego, Calif, USA, 1995.

[2] H. Kressel and J. K. Butler, Semiconductor Lasers and Heterostructure LED's, Academic Press, New York, NY, USA, 1977.

[3] V. L. Kalashnikov, "Mathematical ultra short-pulse laser physics," Maple Application Center-Maplesoft, 2002, http://www .maplesoft.com/applications/view.aspx?SID $=4247$.

[4] W. H. Press, S. A. Teukolsky, W. T. Vetterling, and B. P. Flannery, Numerical Recipes in C: The Art of Scientific Computing, Cambridge University Press, Cambridge, UK, 2nd edition, 1992.

[5] D.-A. Iordache, P. Sterian, F. Pop, and A. R. Sterian, "Complex computer simulations, numerical artifacts, and numerical phenomena," International Journal of Computers, Communications and Control, vol. 5, no. 5, pp. 744-754, 2010.

[6] E. Stefanescu, P. Sterian, and A. Gearba, "Absorption spectra in dissipative two level system media," in Proceedings of the EPS 10 Trends in Physics-10th General Conference of the European Physical Society, 1996.

[7] C. Cattani, S. Chen, and G. Aldashev, "Information and modeling in complexity," Mathematical Problems in Engineering, vol. 2012, Article ID 868413, 3 pages, 2012.

[8] B. A. Malomed, D. Mihalache, F. Wise, and L. Torner, "Spatiotemporal optical solitons," Journal of Optics B, vol. 7, article R53, 2005.

[9] D. Ursescu, O. Tesileanu, D. Balabanski et al., "Extreme light infrastructure nuclear physics (ELI-NP); present status and perspective," in High-Power, High-Energy, and High-Intensity Laser Technology; and Research Using Extreme Light: Entering New Frontiers with Petawatt-Class Lasers, vol. 8780 of Proceedings of SPIE, 2013.

[10] O. Tesileanu, D. Ursescu, R. Dabu, and N. V. Zamfir, "Extreme light infrastructure-nuclear physics," Journal of Physics, vol. 420, Article ID 012157, 2013.
[11] O. Tesileanu, D. M. Filipescu, Gh. Cata-Danil, and N. V. Zamfir, "Nuclear photonics at ELI-NP," Romanian Reports in Physics, vol. 64, supplement, pp. 1373-1379, 2012.

[12] M. E. Crenshaw, M. Scalora, and C. M. Bowden, "Ultrafast intrinsic optical switching in a dense medium of two-level atoms," Physical Review Letters, vol. 68, no. 7, pp. 911-914, 1992.

[13] V. Ninulescu, I. Sterian, and A.-R. Sterian, "Switching effect in dense media," in Advanced Laser Technologies (ALT '01), vol. 4762 of Proceedings of SPIE, pp. 227-234, September 2001.

[14] V. Ninulescu and A.-R. Sterian, "Dynamics of a two-level medium under the action of short optical pulses," in Proceedings of the International Conference on Computational Science and Its Applications (ICCSA '05), vol. 3482, pp. 635-642, Springer, Berlin, Germany, May 2005.

[15] C. Vasile, A. R. Sterian, and V. Ninulescu, "Optically dense media described by help of Maxwell-Bloch equations," Romanian Journal of Optoelectronics, vol. 10, no. 4, pp. 81-86, 2002.

[16] C. Cattani, "Fractional calculus and Shannon wavelet," Mathematical Problems in Engineering, vol. 2012, Article ID 502812, 26 pages, 2012.

[17] S. Chen, Y. Wang, and C. Cattani, "Key issues in modeling of complex 3D structures from video sequences," Mathematical Problems in Engineering, vol. 2012, Article ID 856523, 17 pages, 2012.

[18] H. C. Hsin, T.-Y. Sung, Y.-S. Shieh, and C. Cattani, "A new texture synthesis algorithm based on wavelet packet tree," Mathematical Problems in Engineering, vol. 2012, Article ID 305384, 12 pages, 2012.

[19] B. Mitrica, "Asymmetry of charge ratio for low energetic muons," AIP Conference Proceedings, vol. 972, no. 1, p. 500, 2008.

[20] P. V. Mena, S.-M. Kang, and T. A. DeTemple, "Rate-equationbased laser models with a single solution regime," Journal of Lightwave Technology, vol. 15, no. 4, pp. 717-729, 1997.

[21] B. P. C. Tsou and D. L. Pulfrey, "A versatile SPICE model for quantum-well lasers," IEEE Journal of Quantum Electronics, vol. 33, no. 2, pp. 246-254, 1997.

[22] M. Bulinski and M. L. Pascu, "Chaos in laser diode light emission,” Romanian Journal of Optoelectronics, vol. 9, no. 2, pp. 1-34, 2001.

[23] Y. Liu, N. Kikuchi, and J. Ohtsubo, "Controlling dynamical behavior of a semiconductor laser with external optical feedback," Physical Review E, vol. 51, no. 4, pp. R2697-R2700, 1995.

[24] V. Ninulescu and A. R. Sterian, "Nonlinear dynamics in quantum well laser diodes," in Proceedings of the Europhysics Conference on Computational Physics, Genova, Italy, 2004.

[25] A. R. Sterian, Amplificatoare Optice, Printech Publishing House, Bucharest, Romania, 2006.

[26] R. R. Alfano and S. L. Shapiro, "Observation of self-phase modulation and small-scale filaments in crystals and glasses," Physical Review Letters, vol. 24, no. 11, pp. 592-594, 1970.

[27] A. R. Sterian, V. Ninulescu, and L. Sterian, "Modulated laser diode for medical applications," in 7th Conference on Optics (ROMOPTO '03), vol. 5581 of Proceedings of SPIE, pp. 274-279, September 2003.

[28] D. Marcuse, "Computer simulation of laser photon fluctuations," IEEE Journal of Quantum Electronics, vol. 20, no. 10, pp. 1139-1155, 1984.

[29] D. A. Anghel, A. Rodica Sterian, and P. E. Sterian, "Modeling quantum well lasers," Mathematical Problems in Engineering, vol. 2012, Article ID 736529, 11 pages, 2012. 
[30] V. Ninulescu, V. B. Nicolae, and A. Sterian, "Quantum-well lasers for medical industry," in Proceedings of the Bioinformatics Research and Development (BIRD '08), pp. 563-570, Springer, 2008.

[31] R. Nagarajan, M. Ishikawa, T. Fukushima, R. S. Geels, and J. E. Bowers, "High speed quantum-well lasers and carrier transport effects," IEEE Journal of Quantum Electronics, vol. 28, no. 10, pp. 1990-2008, 1992.

[32] A. R. Sterian, "Coherent radiation generation and amplification in erbium doped systems," in Advances in Optical Amplifiers, P. Urquhart, Ed., InTech, Vienna, Austria, 2011.

[33] F. C. Maciuc, C. I. Stere, and A. R. P. Sterian, "Rate equations for an Erbium laser system, a numerical approach," in Proceedings of the 6th Conference on Optics (ROMOPTO '00), pp. 136-146, September 2000.

[34] F. C. Maciuc, C. I. Stere, and A. R. P. Sterian, "The time evolution and multiple parameters variations in a time dependent numerical model applied for an $\mathrm{Er}^{3+}$ laser system," in Laser Physics and Applications, Proceedings of SPIE, pp. 84-88, September 2000.

[35] A. R. Sterian, "Computer modeling of the coherent optical amplifier and laser systems," in Proceedings of the International Conference on Computational Science and Its Applications (ICCSA '07), vol. 4705 of Lecture Notes in Computer Science, pp. 436-449, 2007.

[36] A. Sterian and V. Ninulescu, "Nonlinear phenomena in erbiumdoped lasers," in Proceedings of the International Conference on Computational Science and Its Applications (ICCSA '05), vol. 3482 of Lecture Notes in Computer Science, pp. 643-650, May 2005.

[37] A.-R. Sterian and V. Ninulescu, "The dynamics of erbiumdoped fiber laser," in Laser Physics and Applications, vol. 5830 of Proceedings of SPIE, pp. 551-555, September 2004.

[38] C. Cattani and I. Bochicchio, "Wavelet analysis of bifurcation in a competition model," in Proceedings of the ICCS, vol. 4488 of Lecture Notes in Computer Science, pp. 990-996, 2007.

[39] A. R. Sterian and F. C. Maciuc, "Numerical model of an EDFA based on rate equations," in Laser Physics and Applications, vol. 5226 of Proceedings of SPIE, pp. 74-78, September 2002.

[40] V. Ninulescu, "Dynamics of a two-mode erbium-doped fiber laser," in Advanced Laser Technologies, vol. 6344 of Proceedings of SPIE, 2006.

[41] F. Sanchez, P. Le Boudec, P.-L. François, and G. Stephan, "Effects of ion pairs on the dynamics of erbium-doped fiber lasers," Physical Review A, vol. 48, no. 3, pp. 2220-2229, 1993.

[42] F. Sanchez, M. LeFlohic, G. M. Stephan, P. LeBoudec, and P.-L. Francois, "Quasi-periodic route to chaos in erbium-doped fiber laser," IEEE Journal of Quantum Electronics, vol. 31, no. 3, pp. 481-488, 1995.

[43] K. Lüdge and H. G. Schuster, Eds., Nonlinear Laser Dynamics: From Quantum Dots to Cryptography, 2011.

[44] J. A. Tenreiro Machado, "Visualizing non-linear control system performance by means of multidimensional scaling," Journal of Computational and Nonlinear Dynamics, vol. 8, Article ID 041017, 7 pages, 2013.

[45] H. G. Winful, "Nonlinear dynamics of semiconductor laser arrays," in Nonlinear Optical Materials and Devices for Photonic Switching, Proceedings of SPIE, pp. 146-149, January 1990.

[46] S. Miclos, I. Lancranjan, R. Savastru, and D. Savastru, "Analysis of bulk and nanostructured passive Q-switches for erbium laser oscillators," in 10th Conference on Optics: Micro- to Nanophotonics III, vol. 8882 of Proceedings of SPIE, 2013.
[47] S. Bennett, C. M. Snowden, and S. Iezekiel, "Nonlinear dynamics in directly modulated multiple-quantum-well laser diodes," IEEE Journal of Quantum Electronics, vol. 33, no. 11, pp. 20762083, 1997.

[48] B. Scott Strachan et al., "Subharmonic resonance cascades in a class of coupled resonators," Journal of Computational and Nonlinear Dynamics, vol. 8, Article ID 041015, 7 pages, 2013.

[49] D. A. Iordache, A. Petrescu, and V. Iordache, "Study of the solitons propagation through optical fibers," UPB Scientific Bulletin A, vol. 72, no. 1, pp. 53-58, 2010.

[50] A. D. Petrescu, A. R. Sterian, and P. E. Sterian, "Solitons propagation in optical fibers computer experiments for students training," in Proceedings of the ICCSA, vol. 4705 of Lecture Notes in Computer Science, pp. 450-461, 2007.

[51] D. Mihalache, "Stable three-dimensional solitons in two-dimensional lattices," in Nonlinear Optics Applications, vol. 5949 of Proceedings of SPIE, pp. 159-168, 2005.

[52] S. V. Demidov and D. G. Levkov, "Soliton-antisoliton pair production in particle collisions," Physical Review Letters, vol. 107, no. 7, Article ID 071601, 2011.

[53] S. Dutta, D. A. Steer, and T. Vachaspati, "Creating kinks from particles," Physical Review Letters, vol. 101, no. 12, Article ID 121601, 2008.

[54] P. N. Velayudhan, "Monopoles and solitons in particle physics [Physics dissertations]," Paper 91, 1983, http://surface.syr .edu/phy_etd/91.

[55] A. Sterian and P. Sterian, "Mathematical models of dissipative systems in quantum engineering," Mathematical Problems in Engineering, vol. 2012, Article ID 347674, 12 pages, 2012.

[56] O. Dănilă, P. E. Sterian, and A. R. Sterian, "Perspectives on entangled nuclear particle pairs generation and manipulation in quantum communication and cryptography systems," Advances in High Energy Physics, vol. 2012, Article ID 801982, 10 pages, 2012.

[57] A. R. Sterian, "The structure of an automatic decision system for a large number of independent particle detectors," Advances in High Energy Physics, vol. 2013, Article ID 839570, 6 pages, 2013.

[58] Y.-H. He, P. Candelas, A. Hanany, A. Lukas, and B. Ovrut, "Computational algebraic geometry in string and gauge theory," Advances in High Energy Physics, vol. 2012, Article ID 431898, 4 pages, 2012.

[59] D. Savastru, S. Dontu, R. Savastru, and A. R. Sterian, "Classic (nonquantic) algorithm for observations and measurements based on statistical strategies of particles fields," Advances in High Energy Physics, vol. 2013, Article ID 876870, 11 pages, 2013.

[60] C. Cattani, "Harmonic wavelets towards the solution of nonlinear PDE," Computers \& Mathematics with Applications, vol. 50, no. 8-9, pp. 1191-1210, 2005.

[61] B. Mitrica, "Energy dependence of the muon charge ratio," $\mathrm{Nu}$ clear Physics B, vol. 151, p. 295, 2006. 

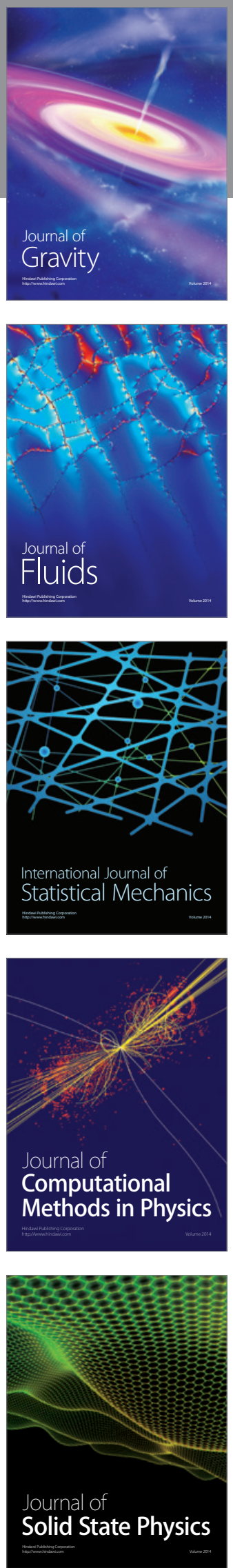

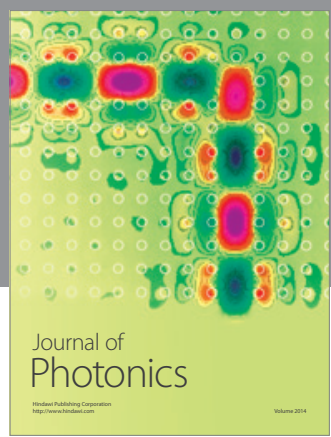

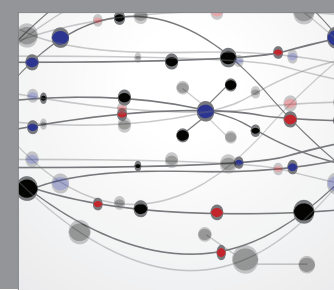

The Scientific World Journal

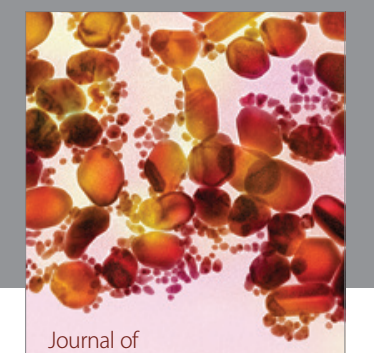

Soft Matter
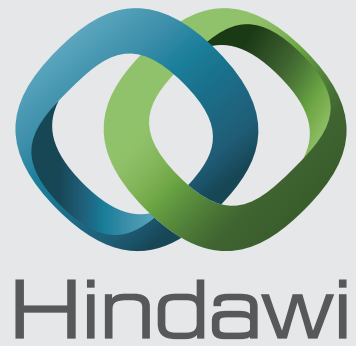

Submit your manuscripts at

http://www.hindawi.com
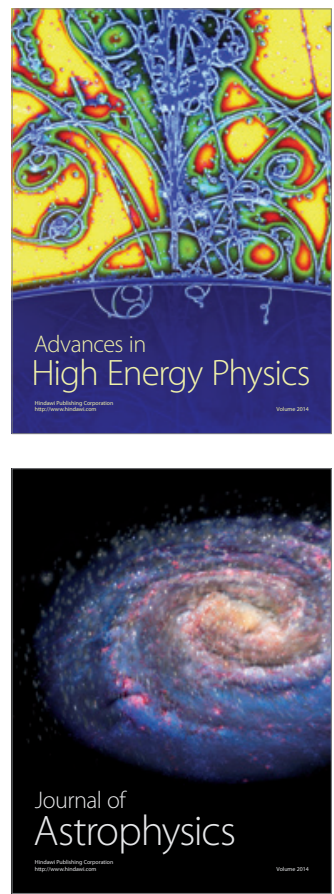
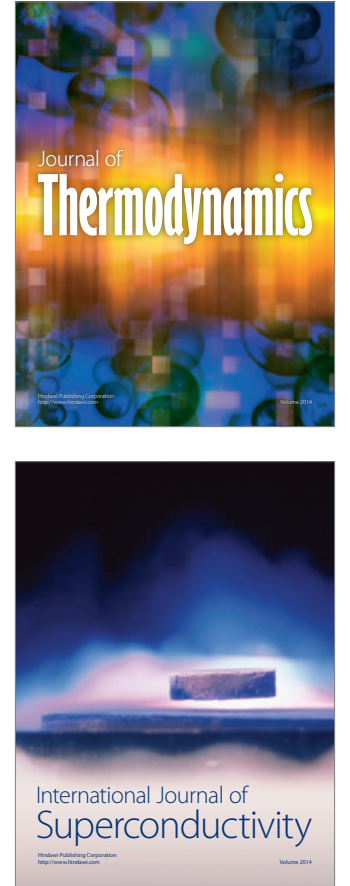
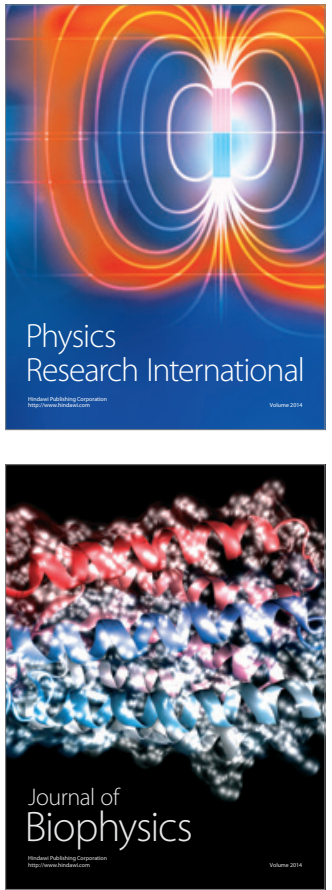
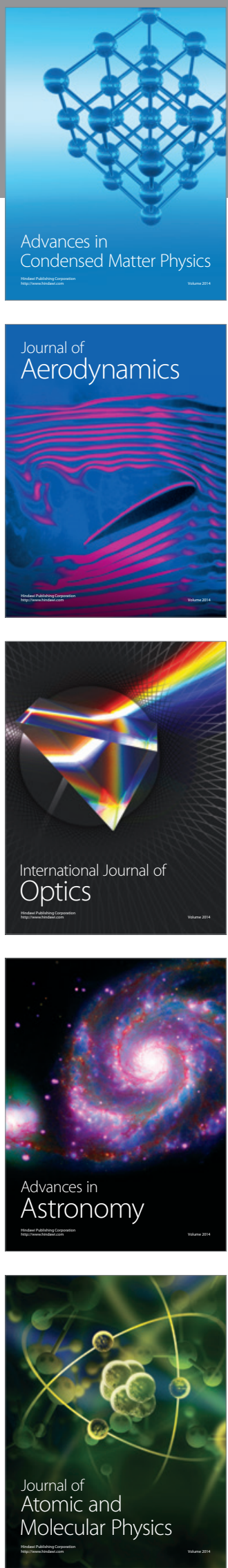